\title{
Réflexions sur la guerre en Islam
}

Reflections on War in Islam

\section{Makram Abbes}

\section{OpenEdition}

\section{Journals}

Édition électronique

URL : http://journals.openedition.org/extremeorient/380

DOI : 10.4000/extremeorient.380

ISSN : 2108-7105

Éditeur

Presses universitaires de Vincennes

\section{Édition imprimée}

Date de publication : 1 novembre 2014

Pagination : 219-233

ISBN : 978-2-84292-414-0

ISSN : 0754-5010

\section{Référence électronique}

Makram Abbes, "Réflexions sur la guerre en Islam », Extrême-Orient Extrême-Occident [En ligne],

38 | 2014, mis en ligne le 01 janvier 2017, consulté le 30 avril 2019. URL : http://

journals.openedition.org/extremeorient/380 ; DOI : 10.4000/extremeorient.380 


\title{
Réflexions sur la guerre en Islam
}

\author{
Makram Abbes
}

\section{Introduction}

Du fait qu'elle soit ramenée à un modèle actuel - le jihad -, la pensée de la guerre en Islam est souvent résumée dans la violence aveugle s'exerçant au nom de préceptes religieux. Tuer au nom de Dieu, vouer une haine inextinguible à l'Autre parce qu'il est différent théologiquement, et penser que le salut de l'âme peut être obtenu par les forfaits honnis de toutes les morales, telle est l'image que certains groupes politiques radicaux ont réussi à propager à propos de la guerre en Islam, et qu'une actualité déchirée par les nombreux conflits n'a cessé de confirmer, y compris chez les spécialistes les plus aguerris et les moins tentés par les essentialismes de tout genre. Si bien qu'affirmer qu'il y a eu d'autres manières de penser la guerre en Islam ou oser des comparaisons avec d'autres cultures relèverait de la gageure, tant les adeptes de cette conception une et indifférenciée de la guerre la confondent avec son identité culturelle et religieuse, à la manière de ceux qui pensent qu'il y aurait une science, une économie, ou une politique, à travers lesquelles se refléteraient les traits d'une identité propre et exclusive. Afin de sortir de cette approche conduisant à de nombreuses impasses, il faut d'abord rappeler le contexte de l'élaboration de la doctrine jihadiste. Cette dernière est, en effet, le produit d'une réaction identitaire globale qui a caractérisé l'époque contemporaine, et qui s'est mise en place, plus précisément, au milieu du $\mathrm{Xx}^{\mathrm{e}}$ siècle avec les théoriciens de l'idéologie islamiste. C'est dans ce contexte de l'extinction du paradigme de l'Empire avec l'abolition de la dynastie ottomane en 1922, et de la délégitimation des modèles politiques anciens au profit de l'État-nation importé d'Europe, qu'une forte pensée réactionnaire et conservatrice émerge dans le sillage des Frères musulmans fondés en Égypte pour proposer une alternative à la culture libérale qui s'est implantée dans l'Orient arabe, et qui a conduit à une modernisation rapide du droit, du statut 
de la femme, des lettres, des lois et des pratiques politiques. L'assomption de cette pensée n'est pas immédiate: elle est contenue dans les années 1950-1960 par les espoirs de la fondation d'un État séculier et moderne, et par la présence d'une culture héritière de la période de la renaissance arabe (la Nahda) qui s'est étalée sur un siècle (milieu du XIX ${ }^{\mathrm{e}}$ siècle-milieu du $\mathrm{XX}^{\mathrm{e}}$ siècle). Mais les échecs répétés lors des conflits avec Israël, la révolution iranienne de 1979 qui présentait un caractère religieux assez prononcé, enfin le contexte géopolitique et économique amenant les Américains à maintenir leur contrôle sur tous les pays de la région, ont fortifié cette tendance guerrière jihadiste avec le modèle théologique dont elle se réclame, et favorisé la confusion entre ses propres pratiques guerrières et l'identité «islamique» qu'elle promeut.

Pour toutes ces raisons, la modernité occidentale est devenue, pour cette tendance, le contraire absolu d'une identité «islamique» souvent mythifiée et fantasmée, et contrairement à certains pays asiatiques qui étaient, au départ, dans la même situation que lui, l'Orient arabe va manquer son rendez-vous avec la modernité. Dans les pays arabo-musulmans, le contexte post-colonial se distingue donc nettement de celui de la Chine et du Japon, avec lesquels ils entretiennent des similitudes étonnantes jusqu'à la fin de la première moitié du $\mathrm{XX}^{\mathrm{e}}$ siècle: présence de la dichotomie entre l'ancien et le moderne, volonté de se moderniser sans perdre son âme, ambivalence du modèle européen qui fascine par ses exploits scientifiques et technologiques, mais inquiète par son impérialisme et la fausse conscience qu'il instaure en associant les valeurs universelles de l'humanisme issues des Lumières à la réalité de sa domination fondée sur la violence et la déstructuration des sociétés colonisées. Pour attirer l'attention sur cette similitude, on peut noter que l'atmosphère culturelle qui a prédominé en Chine lors des guerres de l'Opium correspond globalement à celle que l'Orient arabe va connaître à partir de la campagne de Napoléon en Égypte en 1798. Les belles pages que Damien Morier-Genoud consacre, dans ce dossier, à l'évolution de la conception de l'histoire chez les intellectuels chinois de l'époque, pourraient s'appliquer, à bien des égards, à la période de la Nahda qui a traduit, à sa manière, ce rêve de l'Aufklärung arabe ${ }^{1}$. Cependant, les intellectuels arabes s'interrogent depuis les années 1950, et souvent à travers la comparaison entre l'Orient arabe d'un côté, et la Chine ou le Japon, de l'autre, sur les ratages de la «modernité» arabe aussi bien sur les plans scientifique et économique que du point de vue social et politique. En attendant les réponses qui peinent à émerger dans ce contexte dépressif secoué récemment par les révolutions arabes de 2011, la pensée islamiste continue de

1. Voir, dans ce numéro, l'article de Damien Morier-Genoud: «Écrire l'histoire vis-à-vis de la guerre». 
triompher et de se présenter comme la gardienne de l'identité, seule habilitée à être le truchement de l'histoire et de la religion. Elle est très symptomatique de ces difficultés présentes, de ce malaise profond dans la culture qui ne cesse de paralyser le rapport au présent et au futur, et d'obscurcir le rapport au passé. À tel point que ce rapport est justement devenu l'un des enjeux les plus importants de la lutte contre les lectures qui le prennent à parti, l'hypothèquent en le défigurant et en le gauchissant.

Ce dossier riche et passionnant sur l'art de la guerre en Chine et au Japon nous offre l'occasion de remonter dans le temps pour arriver à une période plus ou moins proche des élaborations antiques qui ont vu le jour en Asie à propos de cet art même si, avec l'Islam, on ne peut reculer au-delà du viI ${ }^{\mathrm{e}}$ siècle. Nous nous proposons ici d'étudier quelques élaborations théoriques de la guerre en Islam et d'attirer l'attention sur la présence de plusieurs types de réflexion, aussi riches les unes que les autres, qui permettent de rejoindre les traditions universelles de la pensée stratégiques, celles de la guerre sainte, de la guerre juste, ou de la guerre en tant que moteur de l'Histoire humaine. Afin d'explorer quelques aspects représentatifs de cet héritage textuel, nous allons aborder la notion de «jihad» qui suscite la polémique, puis nous nous pencherons sur littérature juridique et théologique de la période médiévale; enfin nous attirerons l'attention sur la présence d'une littérature stratégique, fortement liée au genre des Miroirs des princes, qui rejoint, en partie, les maximes militaires représentatives des traditions chinoises.

\section{La notion de «jihad»}

Bien que la notion de jihad ait à l'heure actuelle une signification plus ou moins stable qui gravite autour de la violence armée exercée au nom des préceptes de l'Islam, il n'en reste pas moins qu'elle avait des inflexions sémantiques tout à fait différentes lors de son émergence au vII ${ }^{e}$ siècle, en liaison, notamment, avec le texte coranique. Issue d'une racine renvoyant à l'idée de lutte, de peine et de combat, la notion a très tôt servi à traduire la nouvelle vision de la vie instaurée par le truchement de ce texte, notion par laquelle il poussait les Arabes à adopter une existence fondée sur l'action et le déploiement intense de l'énergie. Il faut être actif à l'échelle individuelle ou sociale, lutter pour un idéal commun, se battre pour des valeurs ou des causes: tel est le premier niveau ontologique extrêmement large que le texte coranique introduit dans la vie des Arabes au $\mathrm{VII}^{\mathrm{e}}$ siècle et qui va permettre d'exalter certaines valeurs universelles hautement prisées, telles que la constance, le courage et la capacité de sublimer ses intérêts individuels. De 
ce point de vue, le contraire du jihad en tant que vision globale de la vie enracinée dans une forte philosophie de l'action est la paresse, l'apathie ou l'indolence. La deuxième signification est plus spécifiquement rattachée à l'idée de lutte, mais il s'agit d'une lutte morale que l'individu engage contre les passions de l'âme. Cet usage s'est répandu avec le développement de la philosophie morale en Islam chez des auteurs comme Ibn al-Muqaffa' (la Grande éthique), al-Mâwardî (les Règles de conduite dans l'ici-bas et dans l'au-delà) ou Miskawayh (la Réforme de l'éthique). Une tradition religieuse attribuée au Prophète désigne ce jihad comme étant le «grand», par opposition à la lutte armée qui serait le «petit jihad». Indépendamment de l'authenticité ou de l'inauthenticité de ce dit, il faut savoir que l'idée de combat spirituel rejoint, au fond, la thématique universelle du gouvernement de soi qui s'est considérablement développée en Islam tant dans les traditions philosophiques que dans les textes religieux ou mystiques. Il faudrait donc se garder de la tendance actuelle qui vise à accorder à la notion de «jihad» un sens purement soufi, et à y réduire les différentes acceptions qu'elle a connues à l'époque médiévale ${ }^{2}$. Tout en partant d'intentions louables visant à combattre la violence armée s'exerçant au nom de la religion, le fait de dire que le «vrai» ou le «bon» jihad correspond à la lutte spirituelle ne reflète pas la réalité des usages linguistiques anciens et modernes, ni ne permet d'aborder sereinement le sens militaire de la notion. En effet, celle-ci a surtout été utilisée par les juristes de l'Islam ainsi que par les penseurs de la guerre comme un synonyme de «harb» qui veut dire guerre tout court et dont le strict contraire est la paix (silm) ou la réconciliation (sulh). Il s'agit là de la troisième signification du jihad qui est d'ordre technique et qui s'est fortement répandue à partir du texte coranique, lequel l'utilise dans de nombreux versets en l'associant à d'autres termes tels que «qital» (combat guerrier) pour décrire les guerres concrètes menées par les premiers musulmans contre leurs ennemis. Ce sens militaire pose de nombreux problèmes d'interprétation car certains estiment qu'il est possible de dégager une signification unique rendant compte de la manière dont le combat armée a été théorisé et conceptualisé dans les textes classiques. Mais cette idée est loin d'être fondée car, dès lors que l'on aborde les aspects juridiques ou stratégiques de la guerre (ou du jihad), apparaissent les divergences de pensée

2. Bien que l'idée de lutte spirituelle soit au cœur du soufisme, il faut savoir qu'elle s'est développée aussi dans les ouvrages d'éthique avec un autre esprit et d'autres finalités qui en faisaient la base de la philosophie politique et de la pensée de la Cité. En raison de son caractère apolitique, voire antipolitique, le soufisme ramène cette idée à l'échelle de l'individu, et l'insère, d'un côté, dans le rapport maître/disciple et, de l'autre, dans les considérations religieuses. 
et d'écoles. Même s'il existe chez les juristes classiques un quasi consensus sur la définition de la guerre en tant que moyen de soumettre les polythéistes à l'Islam, il n'en reste pas moins que de nombreuses différences existent à propos de la désignation de l'ennemi, de la distinction entre combattant et non combattant, du sort des prisonniers de guerre, des règles de partage du butin, de la gestion des terres conquises, sans mentionner bien d'autres points relevant du jus in bello. Par ailleurs, les nombreuses conceptualisations de la guerre, philosophiques (chez al-Fârâbî et Averroès), politiques (chez les auteurs de Miroirs des princes et les spécialistes de la stratégie) ou juridico-théologiques (dans les grandes écoles de droit) empêchent de réduire l'approche de ce thème à l'idée de guerre sainte, de guerre juste, ou à l'acception contemporaine que lui donnent des acteurs et des théologiens issus généralement des mouvements islamistes. Il faudrait donc se garder d'une tendance actuelle dans le champ de la recherche qui tente de démontrer qu'il existe une seule conception de la guerre proprement «islamique». Une telle démarche, qui correspond aux vœux des acteurs se réclamant des textes sacrés de l'islam, ne traduit ni les liens entre la guerre et la politique, ni la manière dont la guerre a effectivement été menée et théorisée en Islam. Par exemple, l'une des plus grandes traditions de réflexion sur la guerre qui était inscrite dans l'art de la stratégie employait indistinctement le terme de «jihad» ou de «harb» dans les différents textes, de même qu'elle prônait des attitudes politiques fondées sur l'importance d'éviter le déclenchement des hostilités par la négociation, la diplomatie ou l'emploi de la ruse. Cette tradition qui refuse le recours à la violence avant d'épuiser les autres formes pacifiques de la résolution des conflits, nous éloigne de la vision guerrière prônée par certains théologiens au nom de la lecture des textes sacrés de l'islam et reprise dans de nombreuses études comme constituant sa marque distinctive. Un autre exemple, puisé dans les traités juridiques dont se réclament indûment les activistes islamistes, montre que la guerre n'était pas, dans les écoles hanafites, shafi'ites ou malikites, une activité arbitraire menée par des individus isolés ou des factions organisées, mais bel et bien un moyen dont disposait l'État et auquel il recourait pour réaliser des objectifs politiques obéissant aux nombreuses formes de rationalités étatiques. À l'opposé de cette conception, le jihad, tel qu'il est pratiqué à l'heure actuelle par de nombreux groupes armés dissidents, vise l'anéantissement de l'État et ne propose, en définitive, aucune vision politique au-delà de l'état de chaos qu'il cherche à instaurer en détruisant les différentes formes d'associations politiques et en tentant de leur substituer des formes religieuses inspirées de leur vision de la Loi (Sharî‘a). 


\section{De la guerre sainte à la guerre juste}

Lors de l'émergence de la tradition juridique au vIII ${ }^{\mathrm{e}}$ siècle, la guerre a fait l'objet d'une forte codification et d'une théorisation à partir des événements historiques de la fondation de l'Islam, et à la faveur de l'intense activité d'exégèse et d'interprétation des textes sacrés. C'est donc à partir de ce croisement entre l'histoire positive (celle des récits de conquête) et les considérations théologico-juridiques (lecture du texte coranique et commentaire de la tradition prophétique) que les grandes lignes du droit de la guerre ont été tracées. L'orientation globale qui marque ce droit consiste à définir le casus belli selon la confession de l'ennemi que l'on affronte. Par ailleurs, du fait que le combat se présente comme la réalisation de la volonté de Dieu et l'application de Sa Loi, il est loisible de parler, en l'occurrence, d'une conception de la guerre analogue à ce qu'on nomme en Occident la «guerre sainte». Au demeurant, la tradition orientaliste identifie majoritairement le «jihâd» à la guerre sainte 3 . Cette traduction ne doit toutefois pas induire une perception erronée de la nature même du pouvoir des chefs qui n'était pas plus divinisé que sacralisé en islam, ni du point de vue des rites, ni au plan des dogmes. De fait, la thématique du pouvoir divin n'a pas été investie comme dans la culture chinoise où le souverain est considéré comme le Fils-du-Ciel (tianzi); on ne saurait même la comparer sur ce point à la culture chrétienne médiévale qui a sacralisé les rois au point de leur attribuer des pouvoirs thaumaturgiques et des vertus quasi divines. Toutefois, le choix de cette traduction par «guerre sainte» pourrait être étayé par le fait que les juristes intègrent le traitement du thème de la guerre dans les devoirs religieux incombant aux croyants, et qu'ils les justifient par des arguments textuels émanant du Coran ou des dits du Prophète. Cette traduction pourrait se justifier également par le fait que l'une des conditions de la validité du combat réside dans la pureté des intentions qui doivent animer le combattant: celui-ci doit partir en guerre en vue de la défense de la religion et «dans la voie de Dieu», comme le stipule l'expression consacrée, et non pas à des fins matérielles (butin) ou personnelles (honneurs, gloire) ${ }^{4}$. Les origines théologiques de la justification de la guerre (défendre la communauté naissante des croyants), de même que la finalité qui lui est assignée (répandre le message de Dieu, soutenir le monothéisme contre le polythéisme) ne laissent donc aucun doute sur le caractère sacré de ce combat. Toutefois, la notion

3. Voir par exemple, Morabia (1993), Flori (2002), Firestone (1999).

4. Voir par exemple le chapitre 3 du livre d'Ibn al-Munâsif, Kitâb al-injâd fì abwâb aljihâd, (2003): 140-141 et Al-Sarakhsî, Sharh kitâb al-siyar al-saghîr dans Al-Mabsût (1989) t. $10: 5$. 
de «guerre sainte», dont les origines remontent à l'Antiquité renvoie aussi à d'autres aspects tels que la sanctification du combat par l'autorité religieuse officielle et la consultation des oracles. «C'est surtout à travers les rituels mis en œuvre, note Bertrand Lafont, que se manifestent en réalité certains aspects "saints" ou "sacrés" des guerres anciennes, ce qui contraste là encore avec la réalité du jihâd où sont principalement en jeu l'accomplissement de la volonté de Dieu et l'ardeur du croyant à combattre l'infidèle, sans que soit mise en avant de conduite rituelle particulière $»^{5}$.

Par ailleurs, la comparaison avec la tradition occidentale de la guerre juste montre que de saint Augustin jusqu'à Vitoria au moins, le combat pour la propagation de la foi et la défense de la cause de Dieu était considéré comme un combat juste, et intégré, de ce fait, dans la réflexion sur la guerre légitime. Chez le premier, «la guerre juste est, par excellence, la guerre inspirée par Dieu, destinée à défendre les communautés qui le craignent» ${ }^{6}$, alors que chez le second, l'opposition à la propagation de la foi chrétienne (qui peut se manifester chez les Indiens d'Amérique par exemple) est une cause de guerre ${ }^{7}$. La conception globale de la doctrine juridique du jihad reste donc proche de la première élaboration de la guerre juste jusqu' $a u \mathrm{XvI}^{\mathrm{e}}$ siècle, dans laquelle l'ennemi est préalablement criminalisé en raison de sa déchéance théologique et par conséquent morale.

Malgré l'importance prépondérante accordée à la dimension religieuse dans la définition du casus belli, la tradition juridique de la guerre en Islam a défini des conditions qui rejoignent les critères de la guerre juste, parmi lesquels nous trouvons notamment la nécessité d'avoir une autorité légitime pour la conduite de la guerre d'un côté, et la distinction entre combattants et non combattants, de l'autre. En ce qui concerne le premier point, il faut noter que le véritable emploi de ces notions dans le droit de la guerre ne se situe pas au niveau du traitement de l'ennemi extérieur, mais plutôt de l'ennemi du pouvoir central représentatif du domaine de l'Islam, c'est-à-dire le dissident ou le rebelle. En effet, lorsqu'il s'agit d'aborder la guerre entre musulmans et les possibilités de l'éclatement d'un conflit interne à la communauté, les juristes sont unanimes à propos de l'emploi de deux expressions: ahl al-'adl (les justes) et ahl-baghiy (les injustes; les rebelles). Affectés par la guerre civile qui a déchiré la communauté quelques décennies après sa naissance (milieu du $\mathrm{VII}^{\mathrm{e}}$ siècle), les juristes s'accordent sur la condamnation de toutes les formes de discorde ou de rébellion qu'ils assimilent à l'injustice suprême. La plupart des

\footnotetext{
5. Lafont (2011): 203.

6. Canto-Sperber (2010): 21.

7. Vitoria (1966): 93-95.
} 
textes sont donc partis du postulat selon lequel les justes et les injustes d'un point de vue moral se confondent, sur le plan politique et militaire, avec les détenteurs du pouvoir d'un côté et les rebelles de l'autre.

L'interdiction de la dissidence et de la rébellion s'est en conséquence accompagnée d'une forte insistance sur la légitimité de l'autorité détentrice du pouvoir. Seule cette autorité est habilitée à lancer la guerre ou à régir des aspects comme le butin, dont les règles de partage sont confiées non pas au chef de l'armée, mais à l'État. Il en va de même pour les prisonniers politiques dont le sort, le plus souvent, est remis aux mains des chefs politiques. Comme dans les textes du Wenzi analysés par Jean Levi, la conception de la guerre juste est tributaire de la souveraineté ${ }^{8}$. C'est en raison de ce lien fort qui unit le caractère juste de la guerre à la conception de la souveraineté que les jihadistes, engagés contre les États installés, refusent en même temps la conception de la souveraineté territoriale et étatique et qu'il la remplacent par la vision théologique d'un pouvoir appartenant uniquement à Dieu (hâkimiyya). Inversement, les juristes de l'islam, fortement travaillés par la défense des pouvoirs souverains, vilipendaient l'insurrection comme une injustice. C'est donc sur le critère de la légitimité du pouvoir que s'opposent, entre autres, les deux types de jihad, ancien et moderne.

D’une manière générale, à partir des années 1950 avec l'affirmation de la pensée islamiste notamment par la voix de l'indo-pakistanais Mawdûdî et de l'égyptien Qutb, la pensée de la guerre épouse une tournure totalement différente de celle que nous rencontrons dans les traités juridiques anciens. Les penseurs de l'islamisme s'appuient en effet sur l'idée que le vrai islam n'a été fondé qu'au vII ${ }^{\mathrm{e}}$ siècle avec le Prophète et ses compagnons, et qu'il convient par conséquence de renouveler cette fondation après plusieurs siècles marqués par l'enfoncement dans l'obscurantisme, l'ignorance et l'impiété. Ainsi, la période de l'islam des origines est-elle considérée comme une utopie créatrice et mobilisatrice, et la représentation de la société du Prophète comme société idéale aboutit, chez ces penseurs, à la barbarisation de la société contemporaine. De ce fait, le jihad s'insère dans une vision de la lutte contre une société barbare et décadente. Les défenseurs de ce modèle radical se réfèrent en général au verset surnommé «âyat al-sayf» (verset du sabre, IX; 5) à partir duquel les juristes de l'époque médiévale ont légitimé le principe de la guerre pérenne contre les impies. Mais en dépit de la réactivation des arguments des Anciens qui accréditent le sentiment de continuité dans la littérature portant sur la guerre, il faut rester averti de la différence qui subsiste dans les rapports

8. Voir, dans ce volume, l'article de Jean Levi: «Morale de la stratégie, stratégie de la morale: le débat chinois sur la guerre juste». 
établis entre la violence et le politique: chez les auteurs classiques, le jihad est considéré comme un instrument aux mains de l'État et la violence des armes est sa seule prérogative. Chez les islamistes, en revanche, le jihad devient l'instrument qui sert à renverser des régimes qualifiés d'impies. Alors que, pour les classiques, la guerre est l'apanage de l'État, pour les islamistes, elle est l'instrument de sa destruction. Par ailleurs, la position des juristes de l'époque médiévale reste extrêmement nuancée en ce qui concerne la désignation de l'ennemi et maintient la distinction entre combattants et non-combattants, ce que le jihadisme moderne remet totalement en cause.

La discrimination entre combattants et non combattants qui est l'une des véritables conquêtes de l'humanisation de la guerre est omniprésente chez les classiques. Pour eux, il y a unanimité sur l'interdiction du meurtre des femmes et des enfants, le point de désaccord portant sur le fait de tuer les moines, les manœuvres, les agriculteurs, les vieillards, les aveugles, les fous et les handicapés. Pour al-Shâfi 'î, et Ibn Hazm, toutes ces personnes (surtout les moines et les travailleurs qui sont des hommes capables de participer à la guerre), doivent être tuées. Pour les autres juristes en revanche (Abû Hanîfa, Mâlik, al-Awzâ'î), cela n'est pas autorisé. Ibn Rushd (Averroès) résume les raisons de cette divergence de la manière suivante: ceux qui estiment que la raison de ces meurtres est l'impiété, comme al-Shâfi'î, ne font aucune distinction au sein des polythéistes ${ }^{9}$, alors que ceux qui fondent leur décision de tuer sur la capacité à combattre ramènent l'interdiction de tuer ces gens à celle qui concerne les femmes qui, a priori, ne prennent pas part au combat. Averroès met en valeur le conflit entre deux arguments provenant du texte coranique :

«Les mois sacrés expirés, tuez les idolâtres partout où vous les trouverez» (IX; 5).

«Combattez dans la voie de Dieu contre ceux qui vous feront la guerre. Mais ne commettez point d'injustice en les attaquant les premiers, car Dieu n'aime point les injustes.» (II; $190^{10}$.)

9. Si al-Shâfîî épargne les femmes et les enfants alors qu’ils sont polythéistes également et de ce fait concernés par le principe selon lequel leurs croyances sont une cause de mise à mort, c'est parce qu'il considère ces deux catégories d'êtres humains comme un bien potentiel pouvant revenir aux musulmans, qu'il est donc interdit de le gaspiller.

10. Le Coran (1970): 59 et 156. En raison d'un classement différent des versets chez Kazimirski, le (II ; 190) devient le (II ; 186). Nous avons opté pour sa traduction parce qu'elle rend bien compte de l'origine du déclenchement de la guerre (agression et riposte à l'agression). 
Averroès commente en disant que ceux qui admettent le principe de l'abrogation d'un verset par un autre pensent que le (IX; 5) a rendu caduc l'effet juridique du (II ; 190) dans la mesure où il lui est historiquement postérieur et qu'il est donc plus à même de révéler le fond de la doctrine islamique en la matière. Ceux qui, au contraire, procèdent d'une autre herméneutique fondée sur la distinction entre la portée générale d'un texte et sa signification particulière estiment que le (IX; 5) n'a qu'une portée générale qui devrait être spécifiée par le (II; 190) ainsi que par tous les versets rappelant qu'une guerre ne se livre que contre des agresseurs ${ }^{11}$. Une telle position change de fond en comble la justification de la guerre, laquelle n'obéit plus à des critères de croyance, mais se trouve ramenée à une posture défensive, l'un des critères fondamentaux de la guerre juste.

\section{La pensée stratégique : prudence, ruse et non violence}

Alors que dans la tradition juridique de l'Islam, le caractère juste d'une guerre est le fruit d'une réglementation intense de tous les aspects relatifs au déroulement du conflit permettant de le rendre légitime et conforme aux enseignements de la Loi, les développements que nous trouvons dans la tradition des Miroirs des princes arabes inaugurent une approche différente de la justice, fondée sur la prudence du prince. Puisant dans le fond politique perse et byzantin, les Miroirs mobilisent aussi bien l'histoire des Anciens que les maximes du bon gouvernement et l'idéal antique du prince juste afin de dégager les grands enseignements sur l'art de la guerre. Leur visée n'est pas tant la fixation normative des lois et la fondation d'un droit de la guerre que la description des qualités du prince et de son comportement idéal en temps de guerre comme en temps de paix. Ici, c'est moins la guerre en tant que telle qui est qualifiée de juste ou d'injuste, que le souverain ou l'État dont il est l'incarnation. Al-Harawî, auteur du XII ${ }^{\mathrm{e}}$ siècle qui a adressé un Miroir à un prince ayyûbide intitulé Mémoire sur les ruses de guerre brosse à grands traits ces exigences éthiques de justice et de lutte contre l'injustice qui représentent l'idéal du prince parfait.

Dieu, dit-il, soutient l'autorité [du prince] pour qu'il abolisse les abus et punisse l'oppresseur, secoure l'affligé et répande les dons, soulage le blessé et libère le

11. Ibn Rushd (Averroès) (1999): 315. Voir la traduction anglaise du passage, The Chapter on Jihad from Averroes'Legal Handbook 'Bidâyat al-mujtahid', in Peters (1977): 1617. 
prisonnier, juge entre l'opprimé et l'oppresseur, distingue entre l'ignorant et le savant, s'efforce avec zèle de défendre l'intégrité de l'Islam et l'ordre du monde. Une fois que le prince connaîtra cette vérité, l'aura bien comprise, y aura réfléchi et s'en sera pénétré, il lui faudra recevoir les faveurs de Dieu avec gratitude et obéissance, en traitant bien ses sujets, en faisant régner la justice, en empêchant l'oppression et la tyrannie, en ordonnant le bien et en interdisant le mal, en faisant observer les commandements de Dieu et en revivifiant la tradition de Son Prophète. [...] Qu'il sache [aussi] que tout se maintient par la justice et se perd par l'oppression, le plus heureux des princes étant celui qui laisse un bon renom et en fait profiter jusqu'à ses successeurs ${ }^{12}$.

Ces lignes inscrivent l'activité militaire dans l'idéal du prince justicier, capable de repousser les agressions, de secourir les victimes de l'injustice et d'aller jusqu'à la défense de l'«ordre du monde». Ici, la moralité s'introduit dans la guerre non pas à travers la normativité du droit, mais grâce à l'éthique de prudence dont doit se munir le prince. Ce fond éthique s'appuie sur deux principes permettant de libérer l'action et de la rendre effective: le naturalisme et le réalisme. En effet, pour des auteurs comme al-Murâdî (De la politique), al- al-Ansârî (De la conduite des guerres) ou al-'Abbâsî (les Traces des Anciens en matière de conservation des États), la guerre est un phénomène universel dont l'essence se situe au-delà de la morale et du droit. Elle est le produit d'une vision de l'homme en tant qu'il est fondamentalement travaillé par les passions de domination et instinctivement soumis à l'«injustice et l'agressivité », selon les termes d'Ibn Khaldûn ${ }^{13}$. De cette anthropologie plus ou moins pessimiste émerge la désillusion quant à la possibilité d'anéantir, en l'homme, ses penchants aux conflits, ou d'espérer faire un jour une dernière guerre qui mettrait fin au cycle de violences qui le tyrannisent depuis toujours. Le mythe d'Armageddon n'anime pas les auteurs des Miroirs, raison pour laquelle le prince doit se préparer continuellement à la guerre, non pas pour livrer la bataille ultime mais parce que, comme chez Machiavel, son métier l'exige. Ainsi, l'horizon théologique d'une guerre qui doit être menée jusqu'à la fin des temps pour anéantir le polythéisme est loin d'être présente chez eux, même si les princes n'hésitaient pas parfois à l'instrumentaliser pour réaliser leurs ambitions de conquêtes territoriales. Cette vision de l'univers politique en tant qu'il est soumis aux vicissitudes du temps et aux accidents de l'histoire pousse le prince à se préparer constamment à la guerre et à en faire l'un de

12. Al-Harawî, Mémoire sur les ruses de guerre, in Sourdel-Thomine (1962): 219. Traduction légèrement modifiée.

13. Ibn Khaldûn (2002): 380 . 
ses plus grands soucis. Le constat de l'existence de la guerre, voire de sa nécessité est lisible à travers la comparaison qu'al- 'Abbâsî engage entre elle et la maladie: la guerre est une réponse aux maux qui affectent le corps politique, pour lesquels on ne saurait trouver de solution pacifique, semblable en cela à l'art de conserver sa santé au moyen de diètes, régimes et remèdes doux. On a donc recours à la guerre une fois épuisées toutes les formes pacifiques de résolution des conflits. Ce n'est donc qu'une fois avéré patent l'échec de tout autre moyen que la guerre se présente comme un dernier recours contre un ennemi récalcitrant resté insensible à l'appel de la sagesse et de la raison. Selon cette vision, la guerre vient sanctionner les attitudes hostiles et belliqueuses d'un ennemi qui a sciemment persisté dans l'erreur.

On reconnaît ici la parenté avec les développements qui ont marqué, en science politique, la tradition réaliste remontant à Thucydide d'autant plus que la tradition des Miroirs, tout comme celle centrée plus spécifiquement sur l'art de la guerre, se sont fortement nourries des traités byzantins ou perses également marqués par l'hellénisme ${ }^{14}$. Mais ce réalisme est loin de déboucher sur une forme de militarisme. Comme dans la pensée byzantine de l'art de la guerre, les auteurs des Miroirs arabes insistent sur le fait que la guerre est une nécessité à laquelle le prince doit se préparer, sans toutefois s'y engager dès que l'occasion lui est offerte. La volonté d'économiser les vies humaines le conduit d'abord à utiliser les moyens diplomatiques et les ruses pour résoudre le conflit: "Évite de chercher la victoire par la rencontre avec l'ennemi, tant que tu trouves le moyen d'utiliser la ruse », affirme al-Harthamî ${ }^{15}$. L'objectif du chef de guerre ne doit être ni le butin, ni la possession des biens matériels, mais l'obéissance de l'ennemi. Or celle-ci peut être obtenue autrement qu'en versant le sang de l'armée adverse, et qu'en exposant au danger ses propres soldats et sa population. Al- 'Abbâsî, quant à lui, va jusqu'à faire de l'observance du principe de la guerre comme ultime recours la qualité suprême du prince : «Le souverain le plus ferme, dit-il, est celui qui ne résout pas les problèmes avec l'ennemi en recourant au combat, tant que d'autres voies lui restent offertes ${ }^{16}$.» La fermeté et la prudence sont les qualités du chef de guerre qui se double nécessairement

14. Outre les Lettres du Pseudo-Aristote à Alexandre qui contiennent de nombreux passages sur la guerre, certains traités byzantins comme le Taktikon d'Elien ou d'autres traités de stratégie rédigés par des anonymes ont probablement été connus à travers une traduction en arabe vers le $\mathrm{VIII}^{\mathrm{e}}$ ou le $\mathrm{IX}^{\mathrm{e}}$ siècle. Pour l'art de la guerre à Byzance, le lecteur peut consulter la synthèse de Jean-Claude Cheynet, in Cheynet (2011) : 231251.

15. Al-Harthamî (1964): 21 .

16. Al-'Abbâsî (1989): 328. 
d'un stratège. Ce dernier se dit mudabbir en langue arabe, terme qui recoupe des significations propres à d'autres champs de savoir : gouvernant en politique, gestionnaire en économie, préparateur de solutions chimique en chimie. Mais le substantif tadbîr qui unit ces différentes significations renvoie à une idée de base, celle de la planification d'une action en fonction de ses fins dernières, tout en veillant à la coordination des différentes étapes de son exécution. Ainsi, la signification linguistique du stratège en arabe qui insiste sur le fait de deviner le futur en sondant le présent, et de penser aux conséquences en ayant les yeux rivés sur les causes se rapproche de celle que l'on trouve dans la maxime du Livre du Prince Shang (Shangjushu) analysée par A. Galvany, et selon laquelle «le sage voit ce qui n'est pas encore en germe», contrairement au sot qui, lui, «n'aperçoit que les faits consommés ${ }^{17}$. Dans le domaine arabe, cette volonté de maîtriser les signes et de trouver une science capable d'aider le prudent dans ses délibérations culmine avec l'introduction de la physiognomonie dans l'art politique et militaire, telle qu'elle est idéalement opérée par le fameux Secret des secrets du pseudo-Aristote. Lire sur les corps les signes qui se dérobent à ceux qui ne savent pas voir, scruter les intentions profondes des individus en contemplant les traits de leurs visages, acquérir un savoir sur l'ami ou l'ennemi par ce simple biais, constituent autant de compétences, réputées secrètes et difficiles à acquérir, que le prince conquérant doit posséder au même titre que les armes de combat.

\section{Conclusion}

N'était l'absence d'une influence certaine de la tradition chinoise de l'art de la guerre sur les textes arabes des Miroirs des princes, on se prendrait à penser que de nombreuses maximes contenues dans ces derniers venaient directement de l'Empire du milieu. Des textes comme le verset 31 du Laozi selon lequel «les armes sont des objets funestes, et ne font pas partie de l'arsenal du sage, qui n'y recourt qu'à son corps défendant» ou l'opposition chère à la période des Royaumes combattants entre la victoire par la langue et celle qui est obtenue par l'épée rappellent le passage de Kalila et Dimna cité plus haut sur l'indispensable usage des stratagèmes avant de se lancer dans le combat. Ces pensées de Chine ancienne font écho au principe évoqué plus haut de ne recourir au combat armé qu'en dernier recours, signe de la plus grand sagesse chez le souverain. Mais si les traces d'une transmission directe ne sont

17. Voir, dans ce numéro, l'article d'Albert Galvany: «Le stratège comme maître des signes: art de la guerre et art sémiotique en Chine ancienne». 
pas avérées, il est possible de penser à des influences indirectes dues à l'afflux continu, dans l'Orient arabe, de populations turques et mongoles, du début de l'Islam jusqu'au moment des conquêtes de Tamerlan au XIv' siècle. Voisins des empires chinois et partageant avec eux de nombreux traits sociaux et culturels, réputés, aussi, pour leurs qualités militaires dès le vIII ${ }^{e}$ siècle ${ }^{18}$, les Turcs deviennent peu à peu les éléments majeurs de l'armée sous les Abbassides, avant de vivre, à leur tour, l'expérience du pouvoir avec les Seljûkides, les Mamelouks ou les Ottomans. Il est fort probable que ces nombreux contacts entre les différentes régions du monde musulman à l'époque médiévale ont permis aux arts, aux techniques et aux idées de voyager et de se transmettre à différents peuples, en Iran, Irak, Syrie, Égypte, Maghreb et Andalousie. Mais il est également possible - abstraction faite des influences directes ou indirectes - que l'expérience historique et la maturité intellectuelle aient produit la même chose à un moment donné de l'histoire, dans des cultures différentes. Cela montre à quel point l'universel est constamment redécouvert par de nombreux biais et revisité par des peuples prêts à vivre la même aventure que leurs prédécesseurs. Ainsi, s'il est certain que les Miroirs des princes ont puisé dans le fond grec (les Lettres d'Aristote à Alexandre), perse (le Testament d'Ardashîr), et indien (Kalila et Dimna), il n'est pas étonnant de constater des parentés avec la tradition chinoise du fait qu'on a pu, dans les deux cultures, penser la guerre à partir de la non guerre. La différence la plus importante à souligner entre elles est que, du côté arabe, il n'existe pas de description des aspects cosmologiques que le stratège doit connaitre et mettre à profit afin de remporter la victoire. Mais les deux se rejoignent dans la volonté d'affirmer la primauté de la ruse et de l'intelligence sur le recours à la force brute: nous sommes en présence de deux courants théoriques qui, bien que distincts dans leurs origines et leurs développements, pensent semblablement la limitation de la guerre à partir de sa reconnaissance en tant que phénomène humain, et se gardent bien de la condamner au nom de principes moraux.

18. Al-Jâhiz (776-867), l'un des grands lettrés de la période abbasside, a composé la Risâla fî manâqib al-turk (Epître sur les qualités des Turcs), dans laquelle il loue notamment leur capacité à manier l'arc à cheval. 


\section{BibliograPHIE}

AL-“ABbÂsî (1989). Âthâr al-uwal fí tartîb al-duwal (les Traces des Anciens en matière de conservation des États). Beyrouth, Dâr al-jîl.

Al-Harawî (1962). Mémoire sur les ruses de guerre. Édité et traduit par Janine SourdelThomine dans «Les conseils du Shayh al-Harawî à un prince ayyûbide». Bulletin d'Etudes Orientales, tome XVII, années 1961-1962. Damas.

Al-Harthamî (1964). Mukhtasar siyâsat al-hurûb, (Abrégé du livre sur la conduite des guerres). Le Caire, al-Mu'assasa 1-misriyya 1-'âmma.

Al-SARAKhSî (1989). Sharh kitâb al-siyar al-saghîr, tome 10, dans Al-Mabsût. Beyrouth, Dâr al-ma'rifa.

Canto-Sperber Monique (2010). L'Idée de guerre juste. Paris, Presses universitaires de France.

Cheynet Jean-Claude (2011). «Légitimer la guerre à Byzance». In La Guerre Juste dans le Proche-Orient ancien médiéval: approches historique, philosophique et juridique. Actes du colloque international tenu à Beyrouth les 29 et 30 mai 2006, publié dans Mélangesde l'Université Saint Joseph, 62.2009 (publié en 2011).

Le Coran (édition de 1970). Traduit par A. Kazimirski. Paris, GF Flammarion.

FIRESTONE Reuven (1999). Jihâd: The Origin of Holy War in Islam. New York/Oxford, Oxford University Press.

FLoRI Jean (2002). Guerre sainte, jihad, croisade. Violence et religion dans le christianisme et l'islam. Paris, Éditions du seuil.

IBn Khaldûn (éd. de 2002). Le Livre des Exemples, I. Autobiographie. Muqaddima. Traduit par A. Cheddadi. Paris, Gallimard.

IBn AL-MunÂSIF (2003). Kitâb al-injâd fî abwâb al-jihâd. Beyrouth, Dâr al-gharb alislâmî.

IBN RUSHD (Averroès) (1999). Bidâyat al-mujtahid wa nihâyat al-muqtasid. Beyrouth, Dâr Ibn Hazm.

LAFONT Bertrand (2011). «Combattre pour son dieu. Aspects religieux de la guerre dans la haute Antiquité proche-orientale». In La Guerre Juste dans le ProcheOrient ancien médiéval : approches historique, philosophique et juridique. Actes du colloque international tenu à Beyrouth les 29 et 30 mai 2006, publié dans Mélangesde l'Université Saint Joseph, 62.

Morabia Alfred (1993). Le Gihâd dans l'Islam médiéval. Le «combat sacré» des origines au XII siècle. Paris, Albin Michel.

Peters Rudolph (1977). Jihad in Mediaeval and Modern Islam. Leiden, Brill.

Sourdel-Thomine Janine (1962). «Les conseils du Shayh al-Harawî à un prince ayyûbide». In Bulletin d'Études Orientales, t. XVII, années 1961-1962. Damas.

Vitoria (de) Francisco (1966). Leçon sur le droit de la guerre. In Leçons sur les Indiens et sur le droit de guerre, Introduction, traduction et notes par M. Barbier. Genève, Librairie Droz: 277-283. 
